\title{
MONITORING CHLOROQUINE RESISTANCE USING PLASMODIUM FALCIPARUM PARASITES ISOLATED FROM WILD MOSQUITOES IN TANZANIA
}

\author{
EMMANUEL A. TEMU,* ISSAC KIMANI, NABUKO TUNO, HITOSHI KAWADA, JAPHET N. MINJAS, AND \\ MASAHIRO TAKAGI \\ Institute of Tropical Medicine, Nagasaki University, Nagasaki, Japan; Department of Parasitology and Medical Entomology, \\ Muhimbili University College of Health Sciences, Dar es Salaam, Tanzania
}

\begin{abstract}
Monitoring antimalarial drug resistance is a useful epidemiologic tool and provides early detection of resistance foci. Using DNA extracted from the head/thorax of wild mosquitoes collected from Bagamoyo Coastal Tanzania, samples infected by Plasmodium falciparum $(N=89$, in 2002 and $N=249$ in 2004) were screened by nested polymerase chain reaction (PCR)-restriction fragment length polymorphism (RFLP) assay for mutations at $P f c r t 76$ and Pfmdr1-86 associated with chloroquine (CQ) resistance. The majority of isolates were of single infection (71\%), and the prevalence of mutant alleles of Pfcrt76 decreased from $64.5 \%$ in 2002 to $16 \%$ in 2004; likewise, mutant Pfmdr1-86 alleles decreased from $46.6 \%$ to $2.7 \%$. Overall, there was a decline of mutant isolates by a factor of 17 and 4 for $P f m d r 1$ and $P f c r t$, respectively. In contrast, isolates with wild-type alleles increased significantly from $<20 \%$ in 2002 to $67.6 \%$ for Pfcrt76 and $83.5 \%$ for Pfmdr1-86 in 2004. This observation suggest a biologic trend of decrease of CQ mutants and a subsequent increase of CQ susceptible parasites in circulation after the discontinued use of CQ in 2001 as a first-line drug in Tanzania. High prevalence of susceptible $P$. falciparum found in circulation not only supports other reports of a decline of mutant parasites after a reduction of drug selection pressure but suggests that the fitness cost is high in mutant parasites. Typing parasite isolates from infected mosquitoes, an alternative means of data collection, has the potential to increase the spatial and temporal coverage, and this approach is practical in highly endemic regions of Africa.
\end{abstract}

\section{INTRODUCTION}

Since the first report of chloroquine (CQ)-resistant Plasmodium falciparum malaria in southeast Asia and South America in the late 1950 s, drug resistance has posed a major problem in malaria control. Today, CQ resistance (CQR) occurs almost everywhere where $P$. falciparum does. After research evidence that indicated that parasites resistant to $\mathrm{CQ}$ and clinical CQ treatment failure rates had reached intolerable levels compared with sulphadoxine-pyrimethamine (SP) and amodiaquine (AQ), governments of many countries changed their treatment guidelines. The Tanzanian government officially changed its malaria treatment policy guidelines, whereby $\mathrm{CQ}$ - the first-line drug for a long time-was replaced with SP in 2001, and recently, to artemisinin-based combination therapy (ACT). ${ }^{1,2}$

CQ resistance in $P$. falciparum is associated with genetic polymorphisms in at least two genes: $P$. falciparum CQR transporter ( $P f c r t)$ located on chromosome 7 and Pfmdr1 encoding the $P$. falciparum P-glycoprotein homologue 1 (Pgh1) located on the parasite chromosomes 5., These genes encode integral membrane proteins localized to the parasite digestive vacuole membrane. ${ }^{4,5}$ One of the mutations at codon 76 of the Pfcrt gene ( $\left.P f_{c} r t 76\right)$, where lysine is replaced by threonine (L76T), has been strongly associated with CQR by parasites and subsequent treatment failure. ${ }^{4}$ At codon 86 of the $P f m d r-1$ gene (Pfmdr1-86), asparginine is replaced with tyrosine (N86Y). This modulates the resistance to parasites harboring the $76 \mathrm{~T}$ mutation, although their role in vivo has not been substantiated. ${ }^{6,7}$ Furthermore, mutations at $P f m d r-1$ may also be associated with resistance to mefloquine and artemisinin, thus highlighting the importance of this gene for the epidemiological study of drug resistance. Additional

* Address correspondence to Emmanuel A. Temu, Department of Vector Ecology and Environment, Institute of Tropical Medicine, Nagasaki University, 1-12-4 Sakamoto, Nagasaki-shi 852-8352, Japan. E-mail: emmatemu@net.nagasaki-u.ca.jp genes may be involved in CQR, because polymorphisms in genes encoding nine other putative transporter proteins have been shown to have significant associations with decreased sensitivity of $P$. falciparum culture-adapted isolates to CQ in vitro. ${ }^{8}$ Even though CQ treatment failure is also affected by other factors such as host immunity and initial parasite load, studies have shown that the two markers, Pfcrt76 and Pfmdr186 , are reliable ${ }^{9}$ and can be used to predict the treatment outcome in malaria-endemic areas. ${ }^{10}$ Recent studies have shown re-emergence of sensitive parasites to $C Q$ after its withdrawal and reduction in the prevalence of mutations associated with CQR. ${ }^{11}$

In conjunction with the conventional in vitro and in vivo drug sensitivity assessments, the use of such molecular markers for early detection of resistance foci and future monitoring of drug-resistant malaria is a useful epidemiologic tool. However, the majority of epidemiological reports on drug resistance are overwhelmingly based on parasite isolates from human clinical cases. In this study, we screened parasite isolates from field-collected mosquitoes for the two mutations, $P f c r t 76$ and Pfmdr1-86, associated with CQR. The mosquitoes were collected from coastal Tanzania in 2002 and 2004, covering the period of 1 and 3 years after the official withdrawal of $\mathrm{CQ}$ as a first-line drug. With the possibility of reintroduction of CQ for treatment of uncomplicated malaria, refining and reviewing our surveillance tools is vital. Molecular typing of isolates from field-collected mosquitoes, an alternative means of data collection, is applicable in high endemic areas such as coastal East Africa, where malaria transmission is intense characterized by high infection rates among vectors.

\section{MATERIALS AND METHODS}

Study site. This study was carried out in Matimbwa and Kongo villages of Bagamoyo (06'30.74 S, 38'55.35 E), located $70 \mathrm{~km}$ north of Dar es Salaam in coastal Tanzania, where malaria is holoendemic. The villages are situated northwest of the Bagamoyo town, in the Yombo administrative division 
with a population of $\sim 21,000$. The district experiences a hot tropical coastal climate and high relative humidity with little variation in annual temperature. The pattern of rainfall is bimodal, with a long period of rain between April and May and a shorter period of rain in October or November. The majority of people in Bagamoyo villages are peasant farmers cultivating cassava, maize, and cashew nuts or have small coconut plantations or rice irrigation farms at subsistence levels. Cattle and goats are common domesticated animals kept around dwellings inhabited by the people. Residential houses are mostly traditionally mud walls and thatched roofs, with very few houses made of cement bricks. P. falciparum is the major malaria parasite accounting for $>95 \%$ of malaria cases in the area. ${ }^{12}$ Both Anopheles gambiae and An. funestus are important vectors in Bagamoyo ${ }^{13}$ and their densities fluctuates following rainfall patterns; consequently, malaria transmission is high and occurs throughout the year. ${ }^{14}$ Informed consent was obtained from the households before the field team accessed their houses.

Specimen's collection and processing. Adult anophelines were sampled fortnightly at five households from April to June in 2002 and 10 households from March to December in 2004. All collections were done inside houses using CDC miniature light traps from 7:00 PM to 6:00 AM, supplemented by early dawn pyrethrum spray catches and indoor house searches by tube aspirators. In the laboratory, Anopheles mosquitoes were identified using morphologic keys, ${ }^{15,16}$ and mosquitoes of An. gambiae and An. funestus groups were included in the final analyses. Specimens were preserved dry in tubes with silica gel and taken to the Institute of Tropical Medicine Nagasaki University in Japan where molecular analysis was undertaken. Because sporozoites, the infective stage of parasites, are located in the salivary glands, genomic DNA from the head and thorax of mosquitoes was processed for molecular analysis. Because loss of polymerase chain reaction (PCR) amplification efficiency is likely to result from inhibitors present in the mosquito tissues, ${ }^{17}$ we used the IsoQuick DNA isolation kit (ORCA Research, Bothell, WA), a silica/guanidinium-based template preparation method, ${ }^{18}$ that efficiently remove PCR inhibitors ${ }^{17}$ on parasite detection from infected mosquitoes.

Determination of mosquitoes infected by parasites. Genomic DNA of the head and thorax was used as a template for a nested PCR assay for identification of samples infected with $P$. falciparum. ${ }^{19}$ Positive and negative controls consisting of $P$. falciparum strain $\mathrm{K} 1$ and master mix without template DNA was used for the 94 samples run. All parasite-infected mosquitoes were subjected to standard multiplex PCR assay to identify respective species belonging to the An. gambiae $^{20}$ and An. funestus ${ }^{21}$ group.

Genotyping of $\boldsymbol{P f} \boldsymbol{c r t}$ and $\boldsymbol{P f m d r} \mathbf{1}$ genes. The nested PCRRFLP assays were used to screen for Pfcrt76 and Pfmdr1-86 genotypes of $P$. falciparum DNA from infected mosquitoes. ${ }^{7,22}$ DNA was amplified using primers flanking residue 76 of the Pfcrt gene in two rounds of PCR. The 145-bp nested PCR product was digested overnight with restriction enzyme ApoI that cuts $P f c r t-76 K$ but not Pfcrt-76T. ${ }^{7}$ Likewise, detection of Pfmdr1 alleles was carried out as described elsewhere. ${ }^{23}$ After the second round of nested PCR, Pfmdr1 alleles at codon 86 were identified by $A p o$ I digestion overnight, which cuts the coding sequence of allele Pfmdr1-86N but not Pfmdr1-86Y. Electrophoresis of each sample, $8 \mu \mathrm{L}$ uncut and
$10 \mu \mathrm{L}$ of restricted digests, was run parallel, each codon at a time, on 3\% (2:1 Metaphor; FMC Bioproducts, Rockland, $\mathrm{ME}$ ) agarose gels stained with $0.5 \mu \mathrm{g} / \mathrm{mL}$ ethidium bromide, with a 100-bp DNA ladder (Fermentas) used to size the bands and visualized under UV. Genomic DNA of $P$. falciparum strains 7G8 (CQ resistant), K1 (CQ sensitive), and 3D7 (CQ sensitive) and a colony An. arabiensis (negative for parasite) maintained in the laboratory were used as positive controls, and $\mathrm{H}_{2} \mathrm{O}$ was used as a negative control.

Data and statistical analyses. In an attempt to evaluate alternative approaches to study the epidemiology of drug resistance, this analysis determined the prevalence of alleles associated with CQR ( $P f c r t 76$ and Pfmdr1-86 genes) among $P$. falciparum isolates from mosquitoes sampled in 2002 and 2004. Absolute numbers were used to calculate the prevalence of different alleles: the mutant (M), wild-types (W), or a combination of mutant/wild-type (WM) infections. Single infection was determined as an isolate with a single genotype; wild-type $P f c r t 76$ and wild-type Pfmdr1-86 (W76W86), mutant Pfcrt76 and mutant Pfmdr1-86 (M76M86), mutant Pfcrt76 and wild-type Pfmdr1-86 (M76W86), or vice versa. Mixed infection was defined as an isolate with more than one genotype; wild-type/mutant Pfcrt76 and wild-type Pfmdr1-86 (WM76W86) or vice versa and wild-type/mutant $P f c r t 76$ and mutant Pfmdr1-86 (WM76M86) or vice versa. A $\chi^{2}$ analysis was performed to compare differences in prevalence of Pfcrt76 and Pfmdr1-86 alleles among vector species and between years of sampling, using EpiInfo, version 3.3 (Centers for Disease Control, Atlanta, GA), and $P<0.05$ was considered significant.

\section{RESULTS}

A total of 89 and 249 mosquitoes infected with $P$. falciparum collected in 2002 and 2004, respectively, were screened for mutations at the Pfcrt and Pfmdrl genes. The mosquito species found and proportion of infected samples screened in 2002 and 2004 were as follows: An. arabiensis (4.5\% versus $21.7 \%$ ), An. gambiae (61.8\% versus $34.4 \%$ ), An. merus $(2.2 \%$ versus $1.3 \%$ ), and $A n$. funestus (31.5\% versus $42.6 \%$ ). At the Pfcrt76 locus, amplification failure was $21.7 \%$ (70/322), much higher than a failure of $7.4 \%(24 / 322)$ at the Pfmdr1-86 locus, suggesting differences in amplification success at the two markers. Failure of amplification might be caused by a low concentration of parasite DNA below the detection level of the nested PCR or differences in the efficiency of amplification obtained by the two assays, with Pfmdr1-86 being marginally more sensitive than that of $P f c r t 76$. Unless stated otherwise, data based on successful PCR amplification are presented from here onward.

The distribution of different alleles at Pfcrt76 and Pfmdr186 observed in 2002 and 2004, among parasite isolates from different mosquito species, indicate a general reduction of mutants and an increase in wild-type isolates (Table 1). The prevalence of parasite isolates with mutant alleles were higher in 2002 than 2004: $64.5 \%$ versus $16 \%$, which is a decrease factor of 4.03 for $P f c r t 76(P<0.0001)$, and $46.6 \%$ versus $2.7 \%$, which is a decrease factor of 17.25 for $P f m d r 1-86(P<0.0001)$. When genotypes with mutant alleles at both $P f c r t 76$ and Pfmdr1-86 (double mutations) were considered, the prevalence of mutant parasites were reduced by a factor of 19.8 , from $59.5 \%(50 / 84)$ in 2002 to $3 \%$ in $2004(7 / 238 ; P<0.001)$. 
TABLE 1

Distribution of wild-type, mutants, and mixed infections of $P f c r t 76$ and $P f m d r 1-86$ genotypes among $P$. falciparum parasites isolates from various vector mosquitoes from Bagamoyo, coastal Tanzania

\begin{tabular}{|c|c|c|c|c|c|c|c|c|c|}
\hline & \multicolumn{3}{|c|}{ Pfcrt 76} & \multicolumn{3}{|c|}{ Pfmdr1-86 } & \multicolumn{3}{|c|}{ Pfcrt76 and Pfmdr1-86 combined } \\
\hline & Mutant & Wild-type & Mixed & Mutant & Wild-type & Mixed & Mutant & Wild-type & Mixed \\
\hline \multicolumn{10}{|l|}{2002} \\
\hline An. arabiensis & & & & & & & & & \\
\hline$(n=4)^{*}$ & 1 & 2 & 0 & 1 & 1 & 1 & 2 & 3 & 1 \\
\hline $\begin{array}{l}\text { An. funestus } \\
\quad(n=28)\end{array}$ & 17 & 5 & 4 & 12 & 5 & 7 & 29 & 10 & 11 \\
\hline $\begin{array}{l}\text { An. gambiae } \\
(n=55)\end{array}$ & 30 & 4 & 12 & 21 & 6 & 17 & 51 & 10 & 29 \\
\hline $\begin{array}{l}\text { An. merus } \\
\quad(n=2)\end{array}$ & 1 & 0 & 0 & 0 & 2 & 0 & 1 & 2 & 0 \\
\hline $\begin{array}{l}\text { Sum of alleles } \\
\quad \text { (\% prevalence) }\end{array}$ & $49(64.5 \%)$ & $11(14.5 \%)$ & $16(21.0 \%)$ & $34(46.6 \%)$ & $14(19.2 \%)$ & $25(34.2 \%)$ & $83(55.7 \%)$ & $25(16.8 \%)$ & $41(27.5 \%)$ \\
\hline $\begin{array}{l}\text { Mean per isolate } \\
(N=76 / 73) \dagger\end{array}$ & 0.645 & 0.145 & 0.210 & 0.466 & 0.192 & 0.384 & 0.557 & 0.168 & 0.275 \\
\hline \multicolumn{10}{|l|}{2004} \\
\hline $\begin{array}{l}\text { An. arabiensis } \\
(n=53) \\
\text { An. funestus }\end{array}$ & 6 & 27 & 7 & 1 & 39 & 6 & 7 & 66 & 13 \\
\hline $\begin{array}{r}(n=104) \\
\text { An. gambiae }\end{array}$ & 14 & 48 & 15 & 2 & 88 & 8 & 16 & 136 & 23 \\
\hline $\begin{array}{c}(n=84) \\
\text { An. merus }\end{array}$ & 7 & 41 & 7 & 3 & 58 & 15 & 10 & 99 & 22 \\
\hline $\begin{array}{l}\quad(n=3) \\
\text { Sum of alleles }\end{array}$ & 1 & 2 & 0 & 0 & 3 & 0 & 1 & 5 & 0 \\
\hline $\begin{array}{l}\text { Sum of alleles } \\
\text { (\% prevalence) }\end{array}$ & $28(16.0 \%)$ & $118(67.4 \%)$ & $29(16.6 \%)$ & $6(2.7 \%)$ & $188(83.4 \%)$ & $29(12.9 \%)$ & $34(8.5 \%)$ & $306(76.9 \%)$ & $58(14.6 \%)$ \\
\hline$(N=175 / 224) \dagger$ & 0.16 & 0.674 & 0.166 & 0.027 & 0.839 & 0.129 & 0.085 & 0.767 & 0.145 \\
\hline
\end{tabular}

$\dagger N=\mathrm{x} / \mathrm{y}$ the total number of infected mosquitoes successfully typed for parasite at Pfcrt76 (X) and Pfmdrt1 $86(\mathrm{Y})$ markers.

There were more isolates with both mutant and wild-type alleles (mixed infection) in 2002 than 2004, 21\% versus $16.6 \%$, which is a decline factor of 1.3 for $P f c r t 76(P=0.4)$, $34.2 \%$ versus $12.9 \%$, which is a decline factor of 2.6 for Pfmdr1-86 $(P<0.0001)$, and $27.5 \%$ versus $14.5 \%$, which is a decline factor of 2 for pooled results $\left(\chi^{2}=12.3, P=0.0004\right.$; Table 1; Figure 1). Overall, the proportion of single infections increased from $72.5 \%(108 / 149)$ in 2002 to $85.5 \%(341 / 399)$ in $2004(P<0.001)$.

Furthermore, when isolates with mixed alleles were pooled together with mutants, the same pattern of decline of mutant parasite was observed. The prevalence of all isolates with mutant alleles and single and mixed infections combined were higher in 2002 than 2004: 85.5\% $(49+16 / 76)$ versus 32.6\% (57/175), a 2.5-fold reduction for Pfcrt $(P<0.0001)$, and $80.8 \%(59 / 73)$ versus $20 \%(35 / 175)$, a 4 -fold reduction for Pfmdr $(P<0.0001)$. At both markers, the prevalence of isolates with mutant alleles inclusive of mixed infection was reduced by a factor of 3.6, from $83.2 \%$ (124/149) in 2002 to $23.1 \%(92 / 399)$ in 2004 (Table 1).

In contrast, there were more wild-type isolates detected in 2004 than 2002: $67.6 \%$ versus $14.5 \%$ for $P f c r t 76(P<0.0001)$ and $83.9 \%$ versus $19.2 \%$ for $P f m d r 1-86(P<0.0001)$. Mutant alleles for both $P f c r t 76$ and $P f m d r 1-86$ combined was significantly reduced by a factor of 5 , from $55.7 \%$ in 2002 to $11.03 \%$ in 2004, whereas isolates with wild-type alleles increased by 4.6 from $16.8 \%$ in 2002 to $76.7 \%$ in 2004 (Table 1; Figure 1).

The above analyses indicate a progressive trend of decline of CQ resistance and an increase of CQ-susceptible parasites observed in a span of 2 years (2002 versus 2004), 1 and 3 years after official withdrawal of CQ as a first-line anti-malaria drug in Tanzania.

\section{DISCUSSION}

Malaria transmission in coastal Tanzania is intense and occurs throughout the year. Over the course of 2 years $(2002$ and 2004), covering a period of 1 and 3 years after the official withdraw of CQ as a first-line drug in Tanzania, we noted a dramatic decrease in frequencies of mutants and accelerated increase of wild-type alleles at Pfcrt76 and Pfmdr1-86 loci among $P$. falciparum isolates from field collected mosquitoes in rural Bagamoyo areas. The decrease in resistant and subsequently increase of susceptible parasites in circulation is consistent with changes in anti-malarial deployment policy in 2001 and the switch to SP after frequent failure of CQ in the country. ${ }^{24}$

A significant decrease of mutant alleles and subsequent increase of susceptible alleles in circulation reported here might be explained by the dramatic reduction of residual drug-resistant parasites that prevailed in 2002, caused by the strong drug pressure imposed before 2001 when CQ was the first-line drug, followed by lower fitness of these resistant parasites, compared with re-emergence of sensitive parasites noted in 2004, in the absence of drug pressure. A striking re-emergence of CQ susceptible parasite observed support the hypothesis that drug-resistant $P$. falciparum parasite may be at competitive disadvantage when drug pressure is removed in agreement with recent epidemiological reports from Malawi, ${ }^{11,25,26}$ Sudan, $^{27}$ and Southeast Asia. ${ }^{28,29}$ Indeed, a 


\section{A: Pfcrt76}

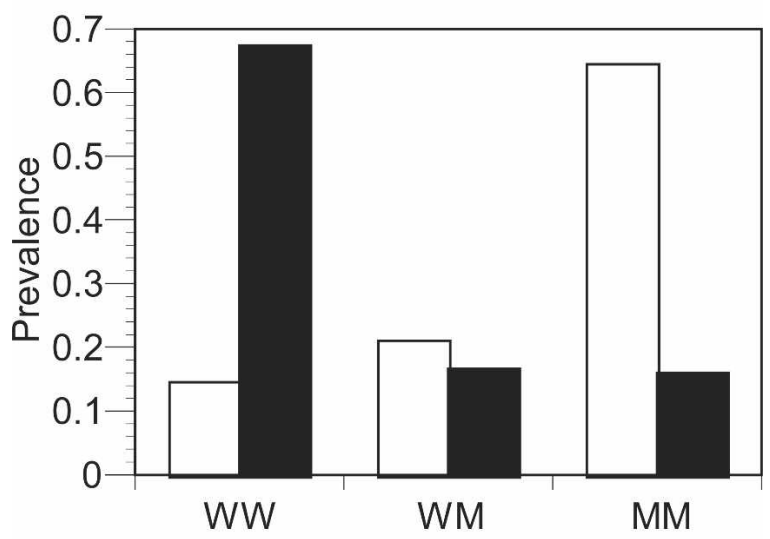

1B: Pfmdr1-86

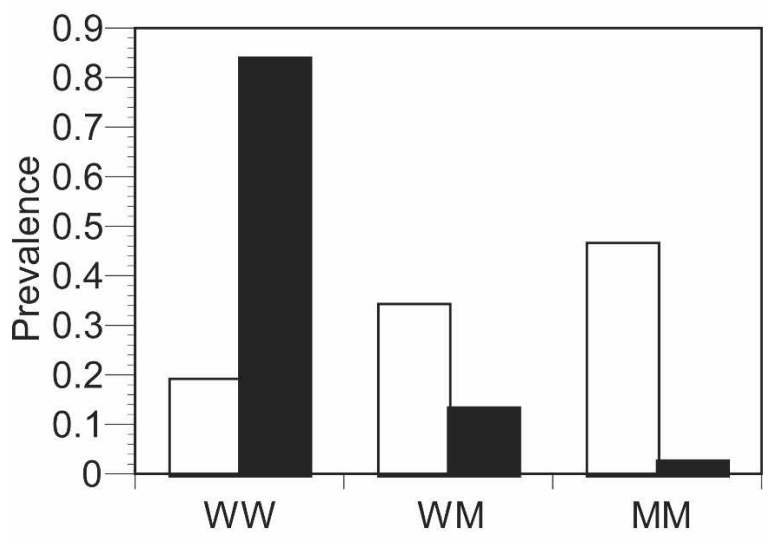

1C: Pfcrt76 \& Pfmdr1-86 combined

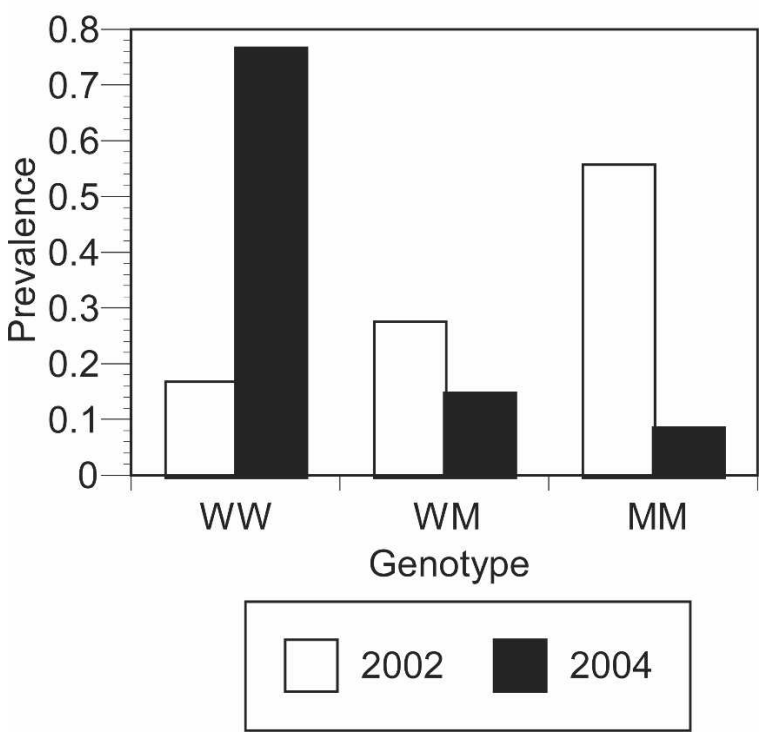

FIGURE 1. Distribution of mutant, wild-type, and mixed alleles of pfcrt76 (A), pfmdr1-86 (B), and both markers combined (C) among parasite isolates from mosquitoes sampled in 2002 and 2004 from Bagamoyo coastal Tanzania. field survey in Malawi, where SP replaced CQ as the first-line drug in 1993, showed that in vitro CQR decreased from $47 \%$ in 1988 to $3 \%$ in 1998 , accompanied by a significant reduction in the prevalence of Pfcrt mutations associated with resistance $^{25}$ attributed to expansion of the wild-type Pfcrt alleles in the parasite population. ${ }^{26}$ In a similar survey, there was an in vitro and in vivo increase in parasite sensitivity to $\mathrm{CQ}$ in Malawi accompanied by significant decreases in the frequency of both CQ-resistant Pfcrt genotype from $85 \%$ in 1992 to $13 \%$ in 2000 and $P f m d r 1$ from $58 \%$ in 1993 to $22 \%$ in $2000 .{ }^{11}$ In our study, the prevalence of mutant alleles at Pfcrt declined by a factor of 4 , from $64.5 \%$ in 2002 to $16 \%$ in 2004 , and those at Pfmdr1 declined by a factor of 17 , from $46.6 \%$ in 2002 to $2.7 \%$ in 2004 . Likewise, a similar pattern of decline of mutant parasites were observed in 2002 and 2004 in analyses involving isolates with 1) mutant alleles at both markers combined $(55.7 \%$ versus $8.5 \%), 2)$ mixed infection alone $(27.5 \%$ versus $14.5 \%$ ), and 3 ) mutant isolates combined with mixed infection and both classified as mutants $(85.5 \%$ versus $32.6 \%)$.

An additional finding of considerable interest is that the rate of decline of parasite isolates with mutant alleles (single and mixed infections combined) was much higher at Pfmdr186 than $P f c r t 76$. Overall, all mutant parasite declined by a factor of 5.2 and 2.6 at $P f m d r 1-86$ and $P f c r t 76$, respectively. A decline of mutant alleles and observed differences in the magnitude of reduction at the two markers suggest variation in fitness burden among parasite with key mutation located at different genes. ${ }^{26}$ Indeed, in vitro studies have shown loss of fitness to the parasite asexual growth rate of the drugresistant forms, in absence of drug pressure ${ }^{30,31}$ and a cost of $25 \%$ caused by mutant alleles at the Pfmdrl gene has been reported. ${ }^{32}$ Likewise, in vivo studies have shown the consequences of fitness cost to transmission, ${ }^{33}$ where resistance is favored under conditions of drug pressure, and in absence of selection pressure against mutants parasites at $P f c r t 76$ and Pfmdr1-86. ${ }^{11}$ In Sudan, a cyclical fluctuation in mutant alleles of $P f c r t 76$ and $P f m d r 1-86$ was observed: higher frequencies were seen during the dry season than during the wet season, reflecting a combination of effect of seasonal variation in drug pressure together with differences in the fitness of resistant and sensitive parasites. $^{27}$

Although a general decline of isolates with mixed infections (i.e., a combination of mutants and wild-type) between 2002 and 2004 was observed, the reduction was only significant at Pfmdrl and when data for both markers were combined. The observed general decline of isolates with mixed infection is consistent with a pattern of decline of CQ mutant parasite after withdrawal of drug selection pressure. It should be noted that, because of the low level of polymorphism at the Pfcrt and Pfmdr genes compared with markers such as merezoite surface protein 1 and 2, current data of mixed infection based on Pfcrt and Pfmdr underestimate the extent of multiplicity of infection among isolates. However, the variation of mixed infections estimated by the two markers might represent a transition period where the frequency of mutants is decreasing and those of susceptible parasites is increasing at different rates coupled by variation in fitness cost inflicted on parasite with mutations at different genes. Also, the detection of mixed infections among isolates could be explained by malaria situation in the area and its vector bionomics. The main vectors in coastal Tanzania, An. gambiae and An. funestus, ${ }^{13}$ 
exhibit strong endophilic and anthropophophagic behavior. ${ }^{34}$ Because multiple blood meals are a normal event among infectious mosquitoes in nature ${ }^{15,35,36}$ and because of the high level of malaria endemicity in the area, the chance of infected mosquitoes becoming re-infected and hyper-infected with parasite is enhanced, and this pattern increases the likelihood of sampling isolates with mixed infection/genotypes as observed in this study.

Among species of malaria vectors in the study area, high proportions of An. gambiae (61.8\% in 2002 versus $34.4 \%$ in 2004) were found infected with parasites for both years, followed by An. funestus (31.5\% versus $42.6 \%$ ). These two Anopheles species are the most efficient malaria vectors in Africa. Although parasite isolates from An. gambiae had high prevalence of mutant alleles in 2002 at both markers compared with other vector species, the same pattern was not observed in 2004, despite the large sample size. As the proportion of resistant parasites decrease in circulation, resulting from reduced drag selection pressure, the same pattern is also reflected among isolates from infected mosquitoes. This indicates random distribution of parasite genotypes per isolate, suggesting lack of association between vector species or selective transmission of mutant alleles of different markers (Pfcrt76/Pfmdr1-86) associated with CQR.

In conclusion, we report a high prevalence of CQ-sensitive $P$. falciparum found in circulation and a pattern of decline of CQ-resistant parasite isolates from field collected mosquitoes, 3 years after the official withdraw of $\mathrm{CQ}$ as the first-line anti-malarial drug in Tanzania. Apart from avoiding rigorous ethical considerations encountered while dealing with humans, typing parasite isolates from infected mosquito has the potential to increase the spatial and temporal coverage; this approach is practical in high endemic regions of Africa. Our results confirm other studies reporting a biological pattern of decline of resistant parasites and subsequent re-emergence of susceptible parasites after drug use is discontinued. Because anti-malarial drug resistance is an ongoing problem, continued monitoring and surveillance is an important aspect of disease management and informs drug policy makers of the possibilities of re-introducing previously withdrawn antimalarial drugs such as CQ in the foreseeable future.

Received May 15, 2006. Accepted for publication September 6, 2006.

Acknowledgments: The authors thank the Bagamoyo village community for allowing us to collect mosquitoes in their houses; the field team of young men from Bagamoyo who were involved in sampling; the Department of Parasitology and Medical Entomology staff, especially S. Kungulilo and Dr D. Gasarasi; the staff of Department of Vector Ecology and Environment for contributing to this study, and Dr M. Alfrangis of CMP Denmark for providing DNA of different strains of parasites used as positive controls in this study. We are grateful to Drs K. Hirayama, A. Kukuchi, and S. Nakazana at the Institute of Tropical Medicine, Nagasaki University, for providing some of the positive controls and valuable technical advice.

Financial support: This study was supported by the JSPS COE to E.A.T.

Disclaimer: The authors declare that they have no competing interests. The funding sources had no role in study design, data collection, analysis, and interpretation. They had no role in writing this report.

Authors' addresses: Emmanuel A Temu, Nabuko Tuno, Hitoshi Kawada, and Masahiro Takagi, Department of Vector Ecology and Environment, Institute of Tropical Medicine, Nagasaki University, 1-12-4 Sakamoto, Nagasaki-shi 852-8523, Japan, Telephone: 95-8497809, Fax: 95-849-7812, E-mail: emmatemu@net.nagasaki-u.ac.jp, tuno@net.nagasaki-u.ac.jp, vergiss@net.nagasaki-u.ac.jp, and mstakagi@net.nagasaki-u.ac.jp. Issac Kimani, Ministry of Health, PO Box 30016, Nairobi, Kenya, Telephone: 254-020-717077, E-mail: isaackimanidr@yahoo.com. Japhet N. Minjas, Department of Parasitology and Medical Entomology, School of Public Health and Social Sciences, Muhimbili University College of Health Sciences, PO Box 65015, Dar es Salaam, Tanzania, Telephone: 255-22-215-3371, Fax: 255-022-215-0465, E-mail: jminjas@muchs.ac.tz.

Reprint requests: Masahiro Takagi, Vector Ecology and Environment, Institute of Tropical Medicine, Nagasaki University, 1-12-4 Sakamoto, Nagasaki-shi 852-8523, Japan. E-mail: mstakagi@net.nagasaki-u.ac.jp.

\section{REFERENCES}

1. Laxminarayan R, 2004. Act now or later? Economics of malaria resistance. Am J Trop Med Hyg 71: 187-195.

2. World Health Organization, 2003. The African Malaria Report. Geneva: World Health Organization.

3. Foote SJ, Kyle DE, Martin RK, Oduola AM, Forsyth K, Kemp DJ, Cowman AF, 1990. Several alleles of the multidrugresistance gene are closely linked to chloroquine resistance in Plasmodium falciparum. Nature 345: 255-258.

4. Fidock DA, Nomura T, Talley AK, Cooper RA, Dzekunov SM, Ferdig MT, Ursos LM, Sidhu AB, Naude B, Deitsch KW, Su XZ, Wootton JC, Roepe PD, Wellems TE, 2000. Mutations in the $P$. falciparum digestive vacuole transmembrane protein PfCRT and evidence for their role in chloroquine resistance. Mol Cell 6: 861-871.

5. Cowman AF, 1991. The P-glycoprotein homologues of Plasmodium falciparum: Are they involved in chloroquine resistance? Parasitol Today 7: 70-76.

6. Reed MB, Saliba KJ, Caruana SR, Kirk K, Cowman AF, 2000. Pgh1 modulates sensitivity and resistance to multiple antimalarials in Plasmodium falciparum. Nature 403: 906-909.

7. Djimde A, Doumbo OK, Cortese JF, Kayentao K, Doumbo S, Diourte Y, Dicko A, Su XZ, Nomura T, Fidock DA, Wellems TE, Plowe CV, Coulibaly D, 2001. A molecular marker for chloroquine-resistant falciparum malaria. $N$ Engl J Med 344: 257-263.

8. Mu J, Ferdig MT, Feng X, Joy DA, Duan J, Furuya T, Subramanian G, Aravind L, Cooper RA, Wootton JC, Xiong M, Su XZ, 2003. Multiple transporters associated with malaria parasite responses to chloroquine and quinine. Mol Microbiol 49: $977-$ 989.

9. Vathsala PG, Pramanik A, Dhanasekaran S, Devi CU, Pillai CR, Subbarao SK, Ghosh SK, Tiwari SN, Sathyanarayan TS, Deshpande PR, Mishra GC, Ranjit MR, Dash AP, Rangarajan PN, Padmanaban G, 2004. Widespread occurrence of the Plasmodium falciparum chloroquine resistance transporter (Pfcrt) gene haplotype SVMNT in $P$. falciparum malaria in India. Am J Trop Med Hyg 70: 256-259.

10. Khalil IF, Alifrangis M, Tarimo DS, Staalso T, Satti GM, Theander TG, Ronn AM, Bygbjerg IC, 2005. The roles of the pfcrt $76 \mathrm{~T}$ and pfmdr1 86Y mutations, immunity and the initial level of parasitaemia, in predicting the outcome of chloroquine treatment in two areas with different transmission intensities. Ann Trop Med Parasitol 99: 441-448.

11. Kublin JG, Cortese JF, Njunju EM, Mukadam RA, Wirima JJ, Kazembe PN, Djimde AA, Kouriba B, Taylor TE, Plowe CV, 2003. Reemergence of chloroquine-sensitive Plasmodium falciparum malaria after cessation of chloroquine use in Malawi. J Infect Dis 187: 1870-1875.

12. Premji Z, Hamisi Y, Shiff C, Minjas J, Lubega P, Makwaya C, 1995. Anaemia and Plasmodium falciparum infections among young children in an holoendemic area, Bagamoyo, Tanzania. Acta Trop 59: 55-64.

13. Temu EA, Minjas JN, Coetzee M, Hunt RH, Shiff CJ, 1998. The role of four anopheline species (Diptera: Culicidae) in malaria transmission in coastal Tanzania. Trans $R$ Soc Trop Med Hyg 92: $152-158$

14. Shiff CJ, Minjas JN, Hall T, Hunt RH, Lyimo S, Davis JR, 1995. Malaria infection potential of anopheline mosquitoes sampled by light trapping indoors in coastal Tanzanian villages. Med Vet Entomol 9: 256-262. 
15. Gillies MT, De Meillon B, 1968. The Anophelinae of A frica South of the Sahara. Johannesburg: South African Institute for Medical Research.

16. Gillies MT, Coetzee M, 1987. Supplement to the Anophelinae of Africa South of the Sahara. Johannesburg: South African Institute for Medical Research.

17. Arez AP, Lopes D, Pinto J, Franco AS, Snounou G, do Rosario VE, 2000. Plasmodium sp.: Optimal protocols for PCR detection of low parasite numbers from mosquito (Anopheles sp.) samples. Exp Parasitol 94: 269-272.

18. Chanteau S, Luquiaud P, Failloux AB, Williams SA, 1994. Detection of Wuchereria bancrofti larvae in pools of mosquitoes by the polymerase chain reaction. Trans R Soc Trop Med Hyg 88: 665-666.

19. Snounou G, Viriyakosol S, Zhu XP, Jarra W, Pinheiro L, do Rosario VE, Thaithong S, Brown KN, 1993. High sensitivity of detection of human malaria parasites by the use of nested polymerase chain reaction. Mol Biochem Parasitol 61: 315-320.

20. Scott JA, Brogdon WG, Collins FH, 1993. Identification of single specimens of the Anopheles gambiae complex by the polymerase chain reaction. Am J Trop Med Hyg 49: 520-529.

21. Koekemoer LL, Kamau L, Hunt RH, Coetzee M, 2002. A cocktail polymerase chain reaction assay to identify members of the Anopheles funestus (Diptera: Culicidae) group. Am J Trop Med Hyg 66: 804-811.

22. von Seidlein L, Duraisingh MT, Drakeley CJ, Bailey R, Greenwood BM, Pinder M, 1997. Polymorphism of the Pfmdr1 gene and chloroquine resistance in Plasmodium falciparum in The Gambia. Trans R Soc Trop Med Hyg 91: 450-453.

23. Duraisingh MT, Jones P, Sambou I, von Seidlein L, Pinder M, Warhurst DC, 2000. The tyrosine-86 allele of the pfmdr1 gene of Plasmodium falciparum is associated with increased sensitivity to the anti-malarials mefloquine and artemisinin. $\mathrm{Mol}$ Biochem Parasitol 108: 13-23.

24. Premji Z, Makwaya C, Minjas JN, 1999. Current clinical efficacy of chloroquine for the treatment of Plasmodium falciparum infections in urban Dar es Salaam, United Republic of Tanzania. Bull World Health Organ 77: 740-744.

25. Mita T, Kaneko A, Lum JK, Bwijo B, Takechi M, Zungu IL, Tsukahara T, Tanabe K, Kobayakawa T, Bjorkman A, 2003. Recovery of chloroquine sensitivity and low prevalence of the Plasmodium falciparum chloroquine resistance transporter gene mutation K76T following the discontinuance of chloroquine use in Malawi. Am J Trop Med Hyg 68: 413-415.

26. Mita T, Kaneko A, Lum JK, Zungu IL, Tsukahara T, Eto H, Kobayakawa T, Bjorkman A, Tanabe K, 2004. Expansion of wild type allele rather than back mutation in pfcrt explains the recent recovery of chloroquine sensitivity of Plasmodium falciparum in Malawi. Mol Biochem Parasitol 135: 159-163.

27. Abdel-Muhsin AM, Mackinnon MJ, Ali E, Nassir el KA, Suleiman S, Ahmed S, Walliker D, Babiker HA, 2004. Evolution of drug-resistance genes in Plasmodium falciparum in an area of seasonal malaria transmission in eastern Sudan. J Infect Dis 189: $1239-1244$.

28. Liu DQ, Liu RJ, Ren DX, Gao DQ, Zhang CY, Qui CP, Cai XZ, Ling CF, Song AH, Tang X, 1995. Changes in the resistance of Plasmodium falciparum to chloroquine in Hainan, China. Bull World Health Organ 73: 483-486.

29. Thaithong S, Suebsaeng L, Rooney W, Beale GH, 1988. Evidence of increased chloroquine sensitivity in Thai isolates of Plasmodium falciparum. Trans $R$ Soc Trop Med Hyg 82: 37-38.

30. Rosario VE, Hall R, Walliker D, Beale GH, 1978. Persistence of drug-resistant malaria parasites. Lancet 1: 185-187.

31. Peters JM, Chen N, Gatton M, Korsinczky M, Fowler EV, Manzetti S, Saul A, Cheng Q, 2002. Mutations in cytochrome b resulting in atovaquone resistance are associated with loss of fitness in Plasmodium falciparum. Antimicrob Agents Chemother 46: 2435-2441.

32. Hayward R, Saliba KJ, Kirk K, 2005. pfmdr1 mutations associated with chloroquine resistance incur a fitness cost in Plasmodium falciparum. Mol Microbiol 55: 1285-1295.

33. Shinondo CJ, Lanners HN, Lowrie RC Jr, Wiser MF, 1994. Effect of pyrimethamine resistance on sporogony in a Plasmodium berghei/Anopheles stephensi model. Exp Parasitol 78: 194-202.

34. White GB, 1974. Anopheles gambiae complex and disease transmission in Africa. Trans R Soc Trop Med Hyg 68: 278-301.

35. Koella JC, Sorensen FL, Anderson RA, 1998. The malaria parasite, Plasmodium falciparum, increases the frequency of multiple feeding of its mosquito vector, Anopheles gambiae. Proc $R$ Soc Lond B Biol Sci 265: 763-768.

36. Briegel H, Horler E, 1993. Multiple blood meals as a reproductive strategy in Anopheles (Diptera: Culicidae). J Med Entomol 30: 975-985. 